CU-TP-663 hep-lat/9412070

\title{
Critical behavior of the chiral condensate at the QCD phase transition *
}

\author{
Shailesh Chandrasekharan ${ }^{\text {a }}$ \\ ${ }^{a}$ Department of Physics, Columbia University, New York, NY 10027, USA
}

We study the critical behavior of the chiral condensate near the QCD phase transition in the background of two fixed light dynamical (sea) quarks. We study the condensate for $5.245 \leq \beta \leq 5.3$ and $10^{-10} \leq m_{\text {val }} \leq 10$ (in lattice units) on a $16^{3} \times 4$ lattice using staggered fermions with $m_{\text {sea }}$ fixed at 0.01 .

\section{INTRODUCTION}

Simulations of the QCD chiral phase transition with dynamical quarks have been carried out in the past for a variety of quark masses at different lattice spacings[1]. For two light flavors of staggered fermions these simulations indicate a smooth transition. If this turns out to be a second order transition, one expects the associated critical behavior to be in the $O(4)$ universality class [2]. There have been attempts[3] to fit the existing data with the $O(4)$ exponents with some success. However to gain confidence in such fits one must understand the physics better, especially in the range of parameters used in these simulations. For example, when the quark masses are sufficiently large, a weak first order signal may not be resolved and could be seen as a smooth transition. In order to answer this question and understand the physics of the transition from a slightly different angle we have asked a somewhat new question; how does the transition look when studied in the small $m_{\text {val }}$ (valence-quark mass) limit, for a small but fixed $m_{\text {sea }}$ (sea-quark mass)?

\section{CHIRAL CONDENSATE}

The order parameter of QCD is the chiral condensate $\langle\bar{\psi} \psi\rangle$ which is given by the formula

$\langle\bar{\psi} \psi\rangle(m, \beta)=\frac{1}{\Omega}\left\langle\operatorname{Tr} \frac{1}{D_{[U]}+m}\right\rangle_{\beta, m}$

*This work was supported in part by the US department of energy.

${ }^{\dagger}$ This work was done in collaboration with D. Chen, N. Christ, R. Mawhinney, W. Lee, and D. Zhu.
Here $D_{[U]}$ is the Dirac operator on the lattice for the gauge configuration $[U]$ and $m$ the quark mass in lattice units. The expectation value is taken over the distribution of gauge configurations given by the usual QCD path integral

$\langle\mathcal{O}\rangle_{\beta, m}=\int d[U] \mathcal{O}[U] \exp \left[-\beta \mathrm{S}_{\mathrm{g}}+\mathrm{S}_{\mathrm{f}}(m)\right]$

where $\mathrm{S}_{\mathrm{g}}$ is the Wilson gauge action with $\beta=$ $6 / g^{2}$ and $\exp \left[\mathrm{S}_{\mathrm{f}}(m)\right]$ is the staggered fermion determinant for two quark flavors of mass $m$. In our convention, $\langle\bar{\psi} \psi\rangle$ is normalized to $1 / m$ for large $m$ by choosing $\Omega$.

\subsection{Partial quenching}

Distinguishing the masses that enter (1) implicitly through the fermion determinant and explicitly through the trace of the propagator, one gets

$\left\langle\bar{\psi} \psi\left(m_{\mathrm{val}}\right)\right\rangle_{\beta, m_{\text {sea }}}=\frac{1}{\Omega}\left\langle\operatorname{Tr} \frac{1}{D_{[U]}+m_{\text {val }}}\right\rangle_{\beta, m_{\text {sea }}}$

In this study we compute $\left\langle\bar{\psi} \psi\left(m_{\text {val }}\right)\right\rangle_{\beta, m_{\text {sea }}}$ for various values of $m_{\mathrm{val}}$ at a small but fixed value of $m_{\text {sea }}$ near the critical coupling $\beta_{c}$. This is relatively easy in comparison to changing the values of $m_{\mathrm{val}}$ and $m_{\text {sea }}$ simultaneously as required in full QCD. However such studies are familiar at zero temperature (far below the critical coupling) and is referred to as the partially-quenched approximation. In this work we have extended such a study to finite temperatures especially to get a better understanding of the phase transition. $\left\langle\bar{\psi} \psi\left(m_{\text {val }}\right)\right\rangle_{\beta, m_{\text {sea }}}$ can be referred to as the partially-quenched chiral condensate. 


\subsection{Dirac spectral density}

If one defines $\rho_{[U]}(\lambda)$ as the density(per unit volume) of eigenvalues of the anti-hermitian Dirac operator $D_{[U]}$ at the eigenvalue $i \lambda$, then one can rewrite (3) as

$\left\langle\bar{\psi} \psi\left(m_{\mathrm{val}}\right)\right\rangle_{\beta, m_{\text {sea }}}=\int_{-\infty}^{\infty} \mathrm{d} \lambda \frac{m_{\mathrm{val}} \bar{\rho}_{\beta, m_{\text {sea }}}(\lambda)}{\lambda^{2}+m_{\mathrm{val}}^{2}}(4)$

where $\bar{\rho}_{\beta, m_{\text {sea }}}(\lambda) \propto\left\langle\rho_{[U]}(\lambda)\right\rangle_{\beta, m_{\text {sea }}}$. In the $m_{\text {val }}=\hat{\xi}$ $m_{\text {sea }} \rightarrow 0$ limit we obtain the familiar Banks $v$ Casher formula [4]

$$
\lim _{m_{\text {val }}=m_{\text {sea }} \rightarrow 0}\left\langle\bar{\psi} \psi\left(m_{\text {val }}\right)\right\rangle_{\beta, m_{\text {sea }}}=\pi \bar{\rho}_{\beta, 0}(0)
$$

When $\bar{\rho}_{\beta, 0}(0) \neq 0$ we have chiral symmetry breaking. Furthermore $\bar{\rho}_{\beta, m_{\text {sea }}}(0)$ is expected to be a regular function of $m_{\text {sea }}$ for small $m_{\text {sea }}$ at least far from the critical coupling where chiral symmetry restoration takes place. Hence we expect $\bar{\rho}_{\beta, m_{\text {sea }}}(0) \neq 0$ below the critical coupling and zero above. This suggests that $\bar{\rho}_{\beta, m_{\text {sea }}}(\lambda)$ will show a striking critical behavior around the critical coupling $\beta_{c}$. This critical behavior can be observed in $\left\langle\bar{\psi} \psi\left(m_{\text {val }}\right)\right\rangle_{\beta, m_{\text {sea }}}$ due to (4).

\section{CRITICAL BEHAVIOR}

In this study we have computed $\left\langle\bar{\psi} \psi\left(m_{\text {val }}\right)\right\rangle_{\beta, m_{\text {sea }}}$ using (3) at a fixed $m_{\text {sea }}=0.01$ in a spatial volume of $16^{3}$ and $\mathrm{N}_{\mathrm{t}}=4$. We have studied $\left\langle\bar{\psi} \psi\left(m_{\text {val }}\right)\right\rangle_{\beta, m_{\text {sea }}}$ for $10^{-10} \leq m_{\text {val }} \leq 10$ which allows us to easily identify the finite volume and finite lattice spacing regions. We have simulated at nine different couplings in the region $5.245 \leq \beta \leq 5.3$. Figure 1 shows some of these results on a log-log plot.

\subsection{Finite lattice effects}

The range of $m_{\mathrm{val}}$ used in our study gives us an idea of the finite lattice-spacing and finite volume effects. We see that for $m_{\mathrm{val}} \geq 0.01$ finite lattice spacing effects become important and for $m_{\mathrm{val}} \leq$ $2.5 \times 10^{-4}$ finite volume effects begin to set it. One knows that in a finite volume for sufficiently small masses the condensate must go linearly to zero. This is convincingly seen in Figure 1 for sufficiently small masses. The region $2.5 \times 10^{-4} \leq$ $m_{\mathrm{val}} \leq 10^{-2}$ could be taken to be free of the finite size and finite lattice spacing effects. This

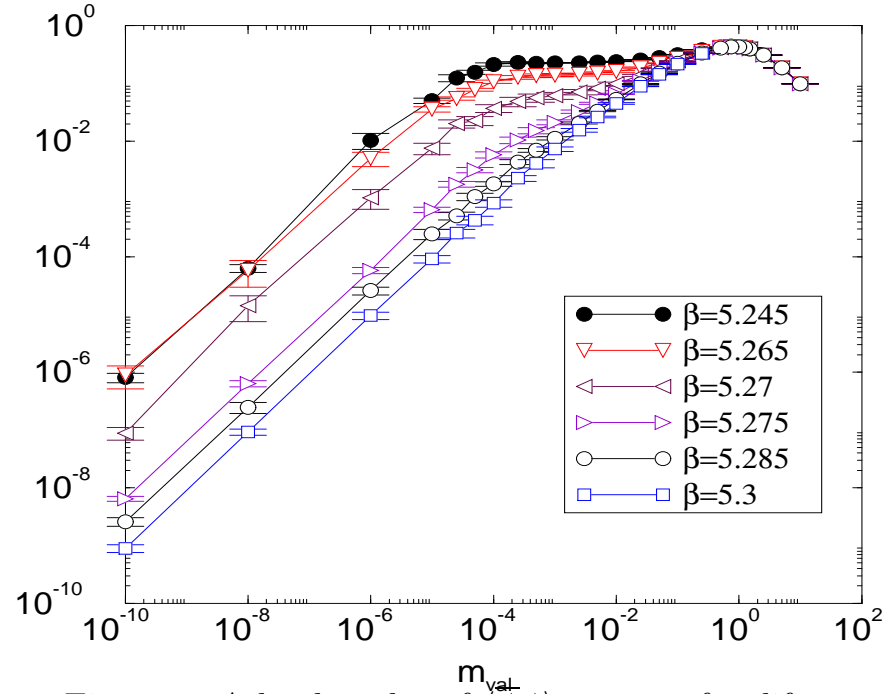

Figure 1. A log-log plot of $\langle\psi \mid \psi\rangle\rangle$ vs. $m_{\text {val }}$ for different values of $\beta$ at a fixed $m_{\text {sea }}=0.01$. The figure clearly shows finite volume and finite lattice spacing effects.

3egionis plotted fireh range of couplings around then critical cipupling on Fighren a pure power law of the form $\langle\bar{\psi} \psi\rangle \sim m^{\frac{1}{\delta}}$ at the critical point. It is interesting to see if such a form also describes the dependence of $\left\langle\bar{\psi} \psi\left(m_{\mathrm{val}}\right)\right\rangle_{\beta, m_{\text {sea }}}$ as $m_{\text {val }} \rightarrow 0$. With the available statistics we find that using a simple least squares fit the data is consistent with a power law over a range of couplings with the power $\frac{1}{\delta}$ ranging from about 0.02 at a $\beta$ of 5.245 to 0.8 at a $\beta$ of 5.3. These fits are shown on Figure 2. The plot of $\langle\bar{\psi} \psi\rangle$ vs. $\beta$ for a range of $m_{\mathrm{val}}$ is given in Figure 3. Here we again see evidence for a smooth transition with the CritfGLUSTiRNSetween 5.26 and 5.275.

The data suggests that at $m_{\text {sea }}=0.01$ there is no discontinuity in the distribution of gauge configurations as a function the coupling. Any possible discontinuity will be enhanced by our method of measuring the condensate at very small valence quark masses. Hence this study supports our earlier results that at $m_{\text {sea }}=0.01$ there is no evidence of a first order transition. Given the accurate results that we have obtained for $m_{\text {sea }}=0.01$ 


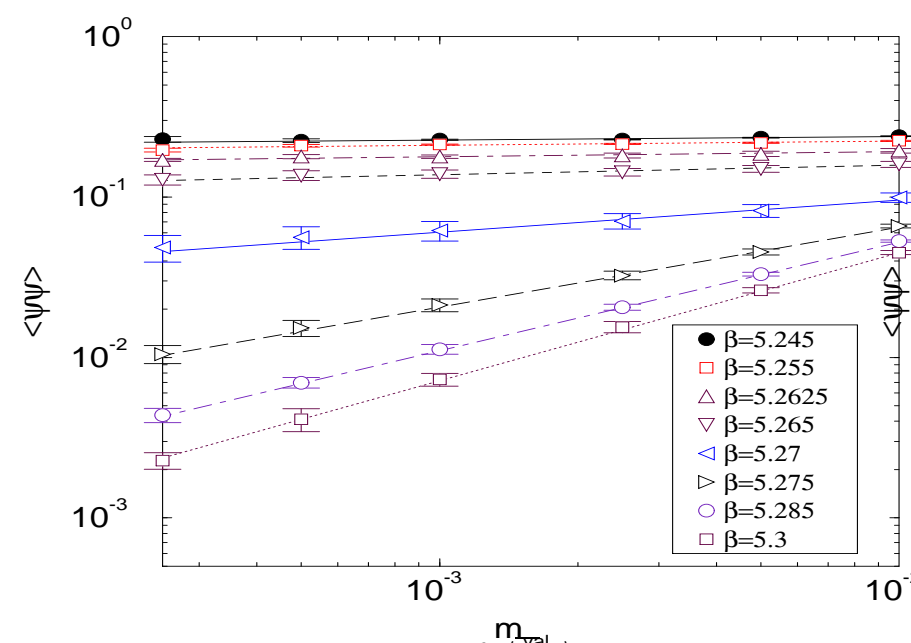

Figure 2. The plot of $\langle\bar{\psi} \psi \vec{\psi}\rangle$ vs. $m_{\mathrm{val}}$ is consistent with power-law behavior over a range of couplings, in the region free of finite lattice effects.

close to the critical region we can compare with our earlier results for $m_{\text {sea }}=0.025$. Figure 1 is a plot of the entropy density from the present run and the earlier run at $m_{\text {sea }}$ of 0.025. Except for a change in $\beta_{c}$ caused by the variation of $m_{\text {sea }}$, the sharpness in the two graphs appear remarkably similar unlike the graphs for $m_{\mathrm{val}}=0.01$ and 0.025 in Figure 3 where $m_{\text {sea }}=0.01$ is fixed. This comparison of Figure 3 and Figure 4 also shows that the sea-quark effects are the dominant effects in the analysis of pseudo-critical coupling done by Karsch [3]. Studying the effects of $m_{\text {sea }}$ and $m_{\text {val }}$ separately will be helpful in understanding some of these observations and can make partially quenched studies a useful technique in learning about the real critical region.

\section{REFERENCES}

1. F. Karsch, Nucl. Phys. B(Proc. Suppl.) 34 (1994) 63 and references therein.

2. K. Rajagopal and F. Wilczek, Nucl. Phys. B399 (1993) 338.

3. F. Karsch, Phys. Rev. D49 (1994) 3791.

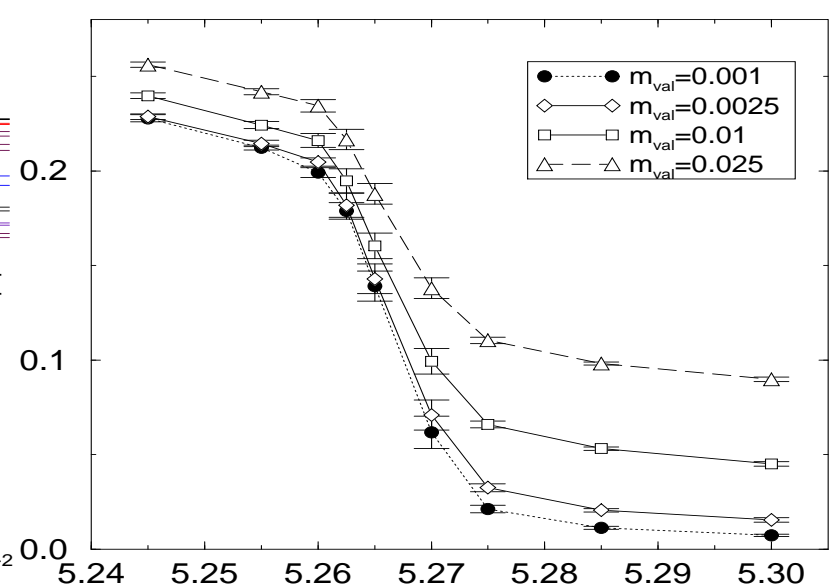

Figure 3. The plot of $\langle\bar{\psi} \psi\rangle$ ss. $\beta$ for various values $m_{\text {val }}$ at a fixed $m_{\text {sea }}=0.01$ shows a smooth transition.

4. T. Banks and A. Casher, Nucl. Phys. B169 (1980) 103 


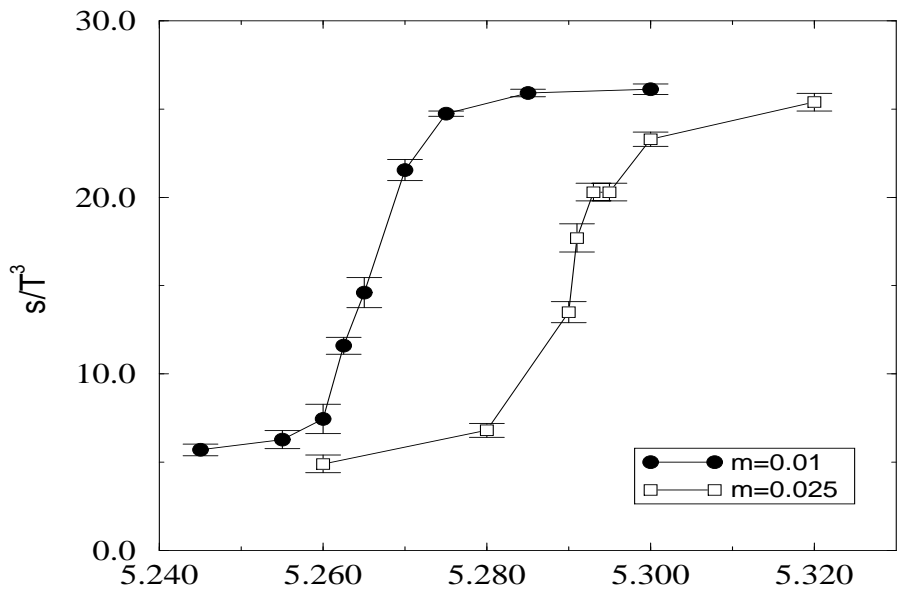

Figure 4. The plot of entrßpy density vs. $\beta$ at $m_{\text {sea }}=m_{\text {val }}$ of 0.01 and 0.025 . 